\title{
From Bowel Obstruction to Perforation: Role of CT as a Troubleshooter Imaging Modality
}

\author{
Manish Kumar ${ }^{1}$, Mayank Jain², Tanuja Sharma ${ }^{3}$, Pramod Kumar ${ }^{4}$, Manjul Mohan ${ }^{5}$ \\ ${ }^{1}$ Assistant Professor, Department of Radiodiagnosis, Rohilkhand Medical College and Hospital, Bareilly, ${ }^{2}$ Junior Resident, \\ Department of Radiodiagnosis, Rohilkhand Medical College and Hospital, Bareilly, ${ }^{3}$ Junior Resident, Department of \\ Radiodiagnosis, Rohilkhand Medical College and Hospital, Bareilly, ${ }^{4}$ Professor, Department of Radiodiagnosis, Rohilkhand \\ Medical College and Hospital, Bareilly, ${ }^{5}$ Assistant Professor, Department of Surgery, Rohilkhand Medical College and Hospital, \\ Bareilly, India
}

Corresponding author: Dr. Mayank Jain, 58, Suraj Nagar, Azadpur, Delhi-110033, India

DOI: http://dx.doi.org/10.21276/ijcmsr.2020.5.1.17

BY-NC-ND

How to cite this article: Manish Kumar, Mayank Jain, Tanuja Sharma, Pramod Kumar, Manjul Mohan. From bowel obstruction to perforation: role of CT as a troubleshooter imaging modality. International Journal of Contemporary Medicine Surgery and Radiology. 2020;5(1):A73-A78.

\section{A B S T R A C T}

Introduction: Bowel obstruction is one of the commonest causes of acute abdomen and emergency surgical admissions. Ultrasonography and abdominal radiography can diagnose bowel obstruction and perforation by revealing dilated intestinal loops and abnormal air-fluid levels in cases of obstruction and extra-luminal air in perforation. However, the exact etiology, site of obstruction and associated complications cannot be properly assessed. The commonest causes of intestinal obstruction are adhesions, external hernias and neoplasms. However, infrequently bowel may get obstructed due to internal hernia, volvulus and encapsulating peritonitis. CT is the most valuable imaging technique for identifying the presence, site and cause of the GI tract perforation. MDCT, by virtue of its excellent multiplanar capabilities \& demonstration of exact anatomical relationships comes to the rescue of radiologists in these grave clinical scenarios. Study aimed at recognizing the imaging features of intestinal obstruction and perforation. Identification of rare causes of bowel obstruction like internal hernia, abdominal cocoon and volvulus.

Assessment of associated complications and differentiation of benign from malignant causes of intestinal obstruction.

Material and methods: This was a prospective study done in the department of Radio diagnosis, Rohilkhand medical college and hospital, Bareilly over a period of two years from July 2017 to June 2019.

Results: The most common cause of obstruction in our study was adhesions followed by external hernias while uncommon causes included volvulus and internal hernias. Majority of gastrointestinal perforations were caused by malignant neoplasms and spontaneous perforations.

Conclusion: MDCT can reliably identify intestinal obstruction and perforation and diagnose its etiology. It holds special importance in identification of rare causes of bowel obstruction such as internal hernia, volvulus and abdominal cocoon. Also, MDCT can reliably identify the site of perforation.

Keywords: Bowel Obstruction,CT, Imaging Modality

\section{INTRODUCTION}

Bowel obstruction is one of the most perplexing clinical situations encountered by a clinician in the emergency room. Small bowel obstruction (SBO) is a common clinical condition that occurs secondary to mechanical or functional obstruction of the small bowel, preventing normal transit of its contents. Bowel obstruction (small/large) either partial or complete is an important cause of morbidity and mortality resulting from acute abdomen.

In a complete or high grade obstruction, there is no fluid/ gas beyond the site of obstruction on delayed scans obtained at 3-24 hours whereas incomplete obstruction shows some fluid /gas beyond the obstruction. In small bowel obstruction, bowel loops are dilated out of proportion to the colon whereas in ileus (observed especially in post-operative patients), there is distension of both colon and small bowel.

Large bowel obstruction (LBO) is four to five times less frequent than $\mathrm{SBO}$ and the causes of $\mathrm{LBO}$ and $\mathrm{SBO}$ differ substantially. The most common finding on a MDCT is presence of a dilated large bowel loop (usually $6 \mathrm{~cm}$, however cecum may be dilated upto $8 \mathrm{~cm}$ ) that gets decompressed distal to transition point. The complications resulting from obstruction might include ischemia, infarction, volvulus and perforation and is frequently seen in strangulated and closed loop obstruction. The most common causes of bowel obstruction includes adhesions, hernias and malignancies. The rare causes of obstruction includes foreign bodies, internal hernias, volvulus, crohn's disease and encapsulating peritoneal sclerosis. Colonic malignancy remains the most common cause of LBO (60\%) followed by sigmoid or cecal volvulus (11-15\%). . $^{1,2,3}$ 
Gastrointestinal (GI) tract perforations can occur due to multifactorial causes with majority of them being emergent situations requiring early recognition and timely surgical treatment. Although air under the diaphragm is a reliable sign of bowel perforation on plain radiography, still CT is most sensitive for detecting free extraluminal, intraperitoneal or retroperitoneal air. In addition to determining the presence of perforation, CT can also localize the perforation site with overall accuracy reported to be in the range between $82 \%$ and $90 \%$.The CT findings of GI tract perforation may be different according to the perforation site, and the various MDCT findings can help predict the perforation site. ${ }^{4}$

Sonography is compatible, low-cost, and easily accessible and attains importance when obstructed bowel segments are dilated and filled with fluid and by using these as a sonic window, the level and sometimes even the cause of obstruction could be reliably identified. On Sonography, bowel obstruction is considered to be present when the lumen of fluid-filled small bowel loops is dilated $>2.5 \mathrm{~cm}$, the length of segment $>10 \mathrm{~cm}$ and peristalsis of dilated segment is increased as shown by the to- $\&$-fro or whirling motion of bowel contents.

Conventional abdominal radiography is the preferred initial radiologic examination and is accurate, widely available and inexpensive. Abdominal radiography, both dependent (supine/prone) and non-dependent (upright/decubitus) can diagnose bowel obstruction and perforation. The different signs pertaining to obstruction are dilated gas/fluid filled small bowel, gasless abdomen, multiple air-fluid levels, airfluid levels longer than $2.5 \mathrm{~cm}$, air-fluid levels in same loop of small bowel of unequal heights. The key radiographic signs that allow distinction between a high-grade SBO and a lowgrade obstruction are the presence of small bowel distention, with maximal dilated loops averaging $36 \mathrm{~mm}$ in diameter and exceeding $50 \%$ of the caliber of the largest visible colon loop as well as a 2.5 times increase in the number of distended loops in the abdomen compared with the normal number.

MDCT because of its rapid identification and increased sensitivity and specificity exhibits a high diagnostic performance over ultrasonography and conventional radiography, especially when the underlying cause is abnormal and has superseded them for more rapid and accurate diagnosis. The presence of intraluminal gas and particulate material in the dilated small bowel loops 'the small bowel feces sign' is abnormal. The 'string of beads sign' is said to be pathognomonic of small bowel obstruction (Table III). Unlike oral contrast radiography, which images only the luminal surface, CT allows imaging of the abdominal contents outside the lumen also. Because of this advantage, the nature of the obstruction can be established. Nonvisualization of oral contrast in the colon 12 hours after its administration is a reliable indicator of complete obstruction on CT whereas its visualization suggests a low- grade or partial obstruction. ${ }^{5}$ One advantage of CT over radiography is ease of identification of transition zone, the site where dilated bowel transitions to decompensated bowel. Further, it also provides an excellent evaluation of bowel wall, its vessels and adjacent mesentery, which permits the identification of co-existent ischemia and /or infarction. MDCT through its multiplanar reformations (MPR) can increase both accuracy and confidence in the location of the transition zone in $\mathrm{CT}$ of SBO. ${ }^{6} \mathrm{CT}$ also provides excellent evaluation for presence of bowel perforation and presence of extraluminal gas.

\section{MATERIAL AND METHODS}

Our study was a single centre prospective study conducted in the department of Radiodiagnosis, Rohilkhand medical college and hospital, Bareilly over a period of two years (July 2017 to June 2019). A total of 100 patients presenting to the department of surgery with suspected intestinal obstruction and/or perforation and who underwent MDCT after preliminary investigations were included in the study.

\section{Inclusion criteria}

Patients diagnosed of having intestinal obstruction and perforation by conventional methods (ultrasonography/XRay abdomen) and who underwent a MDCT based on the final etiological outcome.

\section{Exclusion criteria}

Decompensated patients

Pregnancy

Patients with deranged kidney function tests (KFT)

Post-operative patients

\section{Methods of collection of Data}

After the confirmation of clinical findings and preliminary investigations, conventional techniques such as abdominal radiographs and ultrasonography were done. Patients were then taken for MDCT (Table I). Volumetric data of the abdomen were acquired with MDCT before \& after intravenous contrast administration. Oral administration of contrast was not given in high grade. However, in cases of partial obstruction oral contrast medium was administered 30-120 minutes before scanning. Sagittal, coronal, and curved multiplanar reformations were made. All CT scans were performed on Bright Speed GE 16 slice Elite CT scanner with pitch of $1.75: 1$, section reconstruction interval of $2 \mathrm{~mm}$ and speed $35 \mathrm{~mm} /$ rotation.

\section{RESULTS}

\section{General characteristics}

Out of 100 patients, 64 were males (64\%) and 36 were females $(36 \%)$. There were $32(32 \%)$ patients between the age 15 and 30 years, 27 (27\%) patients between the age 31 and 45 years, $26(26 \%)$ patients between the age 46 and 60

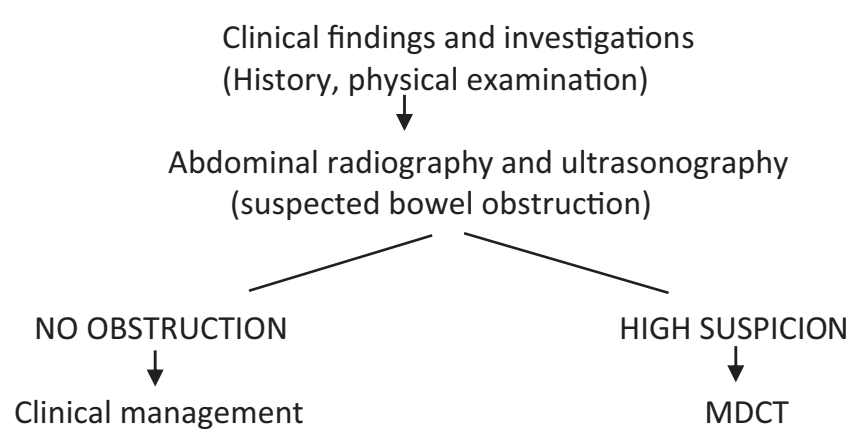

Table-1: Algorithm for diagnostic work-up of patients with suspected bowel obstruction 
(a)

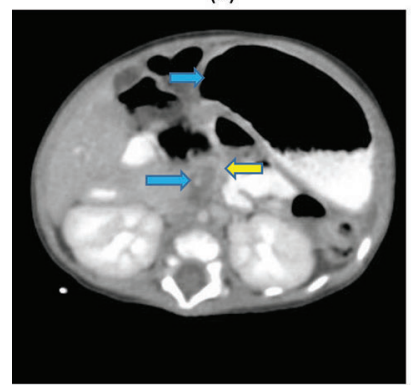

(b)

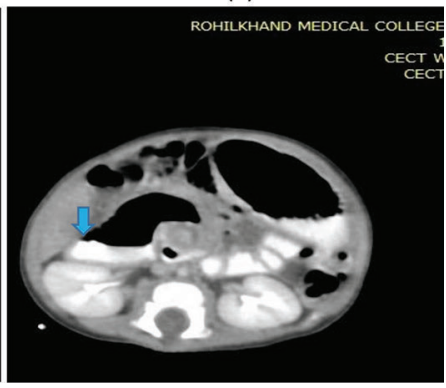

Figure-1: Midgut volvulus. Axial CECT scan (a) shows distension of stomach, Ist, IInd and IIIrd parts of duodenum. Whirlpool appearance of mesenteric vessels with SMV (yellow arrow) overlapping around SMA and vein is noted to the left of SMA. (b) Duodeno-jejunal junction and proximal jejunal loops in right part of abdomen s/o malrotation.

(a)

(b)

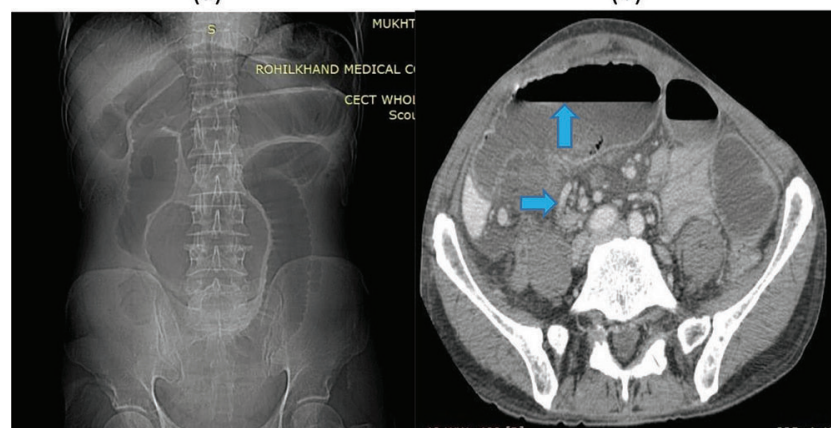

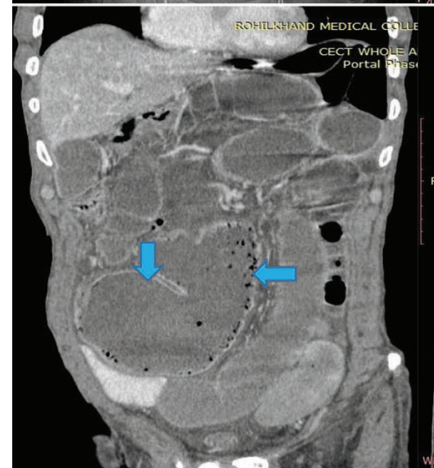

(c)

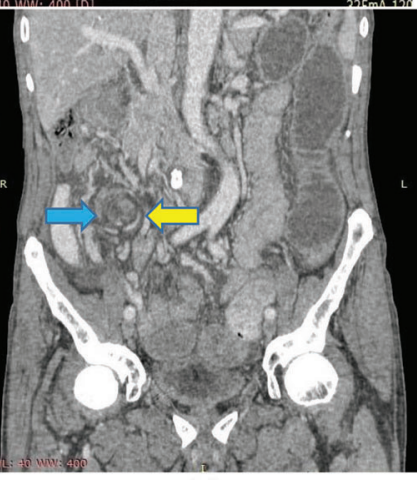

(d)
Figure-2: Cecal volvulus. a) Conventional radiography shows non-specific signs of air filled dilated loops in midabdomen and left upper quadrant. b) Axial scans shows dilated jejunal loops and twisting of vessels and mesentery. c) Coronal scans shows markedly dilated cecum. d) WHIRL SIGN-Twisting of mesentery in right lower quadrant around branch of superior mesenteric artery (SMA) with low attenuating fatty mesentery with enhancing engorged vessels radiate from the twisted bowel. The central soft tissue density (yellow arrow) represents the source of the twist.

years, and $15(15 \%)$ patients above 60 years of age. Out of 100 patients, 24 patients presented with perforation at the time of investigation.

\section{Distribution of clinical symptoms and signs}

All the 100 patients had pain abdomen while distension, vomiting and constipation/obstipation were present in decreasing order.

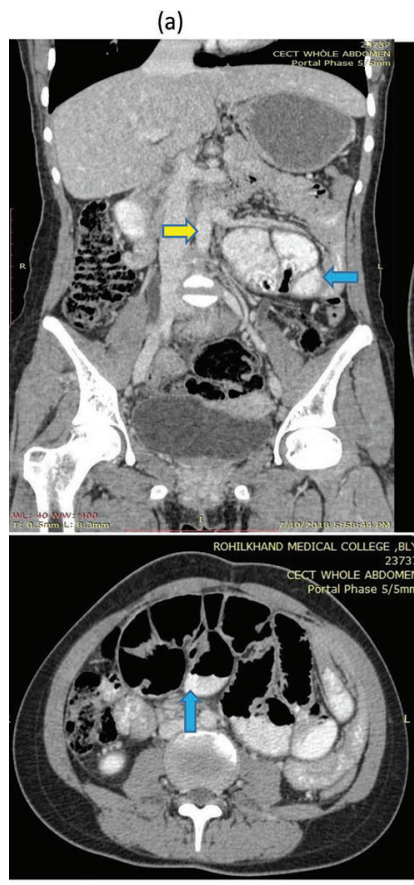

(c) (b)
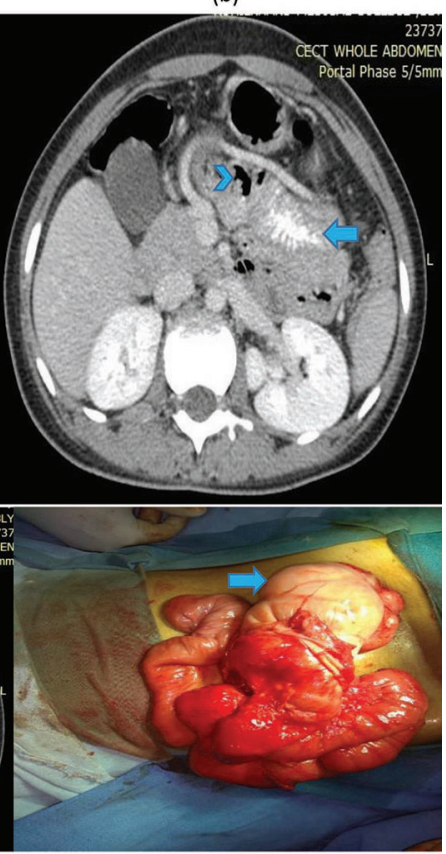

(d)
Figure-3: Internal hernia. a) Coronal scan shows cluster of fluid-filled, distended sac-like intestinal loop in left anterior pararenal space (Closed loop) with inferior mesenteric vein anterior and medial to hernial orifice (yellow arrow) and entrapped intestine. Axial scans (b,c) shows convergence of bowel, mesenteric fat, and vessels of the closed loop alongwith engorgement and twisting of vessels at orifice (Hernial orifice) and dilated bowel loops. d) Intra-op images of the same patient were consistent with the MDCT findings.

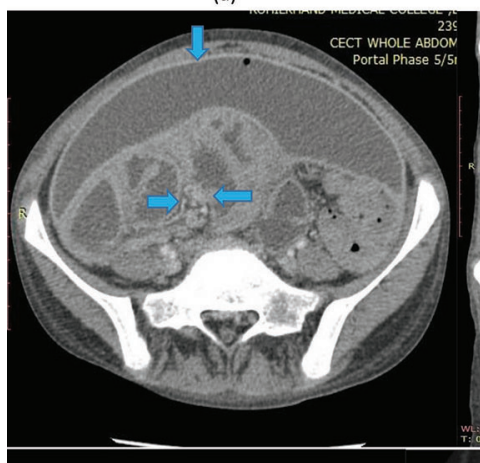

(b)

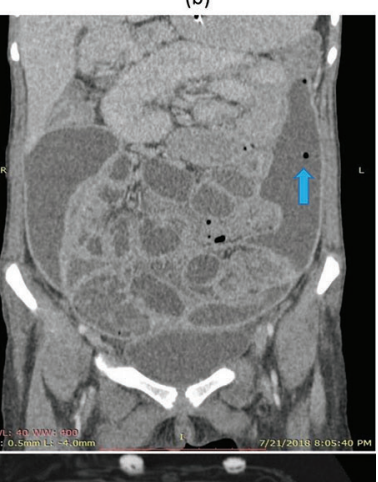

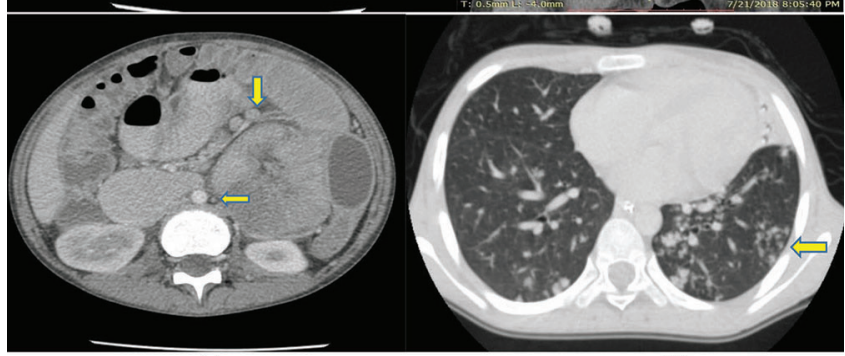

(c) (d)
Figure-4: Abdominal cocoon. Axial (a) and coronal (b) scans show large collection in peritoneal cavity showing thickened $(>2 \mathrm{~mm})$ and enhancing walls and internal air foci and dilated small bowel loops. Axial scans(c, d) show mesenteric and retroperitoneal lymphadenopathy (yellow arrows) and treein-bud appearance in lower portion of lungs. 
(a)

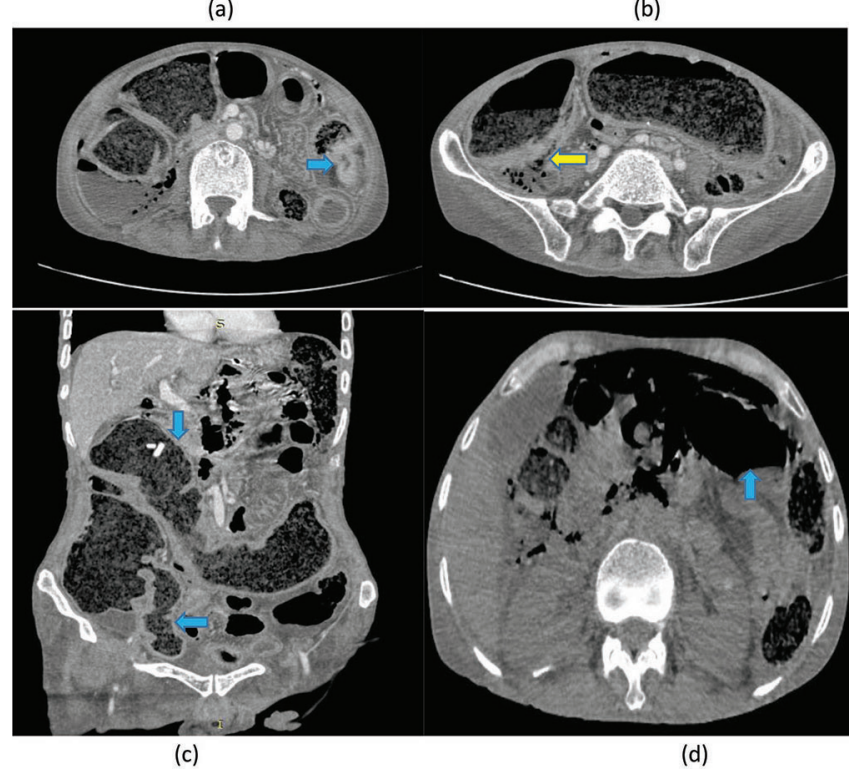

Figure-5: PERFORATION SECONDARY TO NEOPLASM. Axial scans shows concentric wall thickening and enhancement in distal transverse colon (a) with a defect in cecum proximal to ileo-cecal junction (yellow arrow) (b). (c) Coronal scans shows marked fecal loading of large bowel and distal ileum. (d) Pneumoperitoneum.

\begin{tabular}{|l|c|}
\hline Etiology & $\begin{array}{c}\text { No. of } \\
\text { cases }\end{array}$ \\
\hline Adhesions & 25 \\
\hline External hernias & 21 \\
\hline Malignancies & 14 \\
\hline Obstruction secondary to infection/ inflammation & 16 \\
\hline Volvulus & 9 \\
\hline Abdominal cocoon Table-2: Etiology & 4 \\
\hline Intussusception & 7 \\
\hline Internal hernia & 4 \\
\hline \multicolumn{2}{|c|}{} \\
\hline
\end{tabular}

\begin{tabular}{|l|l|}
\hline Site & CT Features of bowel obstruction \\
\hline Small bowel & $\begin{array}{l}\text { Dilated bowel loops }>2.5 \mathrm{~cm} \\
\text { String of beads appearance, Small bowel } \\
\text { feces sign } \\
\text { Signs specific to cause* }\end{array}$ \\
\hline Large bowel & $\begin{array}{l}\text { Dilated bowel loops }>6-8 \mathrm{~cm} \text { (DEPENDING } \\
\text { UPON SITE) } \\
\text { Cecum }-8 \mathrm{~cm} \\
\text { Rest of large bowel loops }-6 \mathrm{~cm} \\
\text { Dilated bowel loops proximal to transition } \\
\text { point \& decompressed bowel distal to it. } \\
\text { Signs specific to cause* }\end{array}$ \\
\hline \multicolumn{2}{|l|}{ Table-3: CT Features of bowel obstruction } \\
\hline
\end{tabular}

\section{Etiology}

In our study, adhesions and hernias are the most common cause of obstruction, accounting for $25 \%$ each. Out of 25,4 patients had internal hernia and rest were obstructed indirect inguinal hernia and obstructed umbilical hernia. Obstruction

\begin{tabular}{|l|}
\hline CT features of GI tract perforation \\
\hline Direct sign \\
-Presence of extraluminal air \\
-Discontinuity of bowel wall \\
\hline Indirect sign \\
-Inflammatory changes adjacent to bowel wall, so called "Dirty \\
fat sign". \\
-Focal bowel wall thickening \\
\hline \multicolumn{1}{|c|}{ Table-4: CT Features of bowel perforation } \\
\hline
\end{tabular}

secondary to infection or inflammation (16\%) such as tuberculosis, meckel's diverticulum was closely followed by malignancies (14\%). The rare causes of obstruction included volvulus (9\%), intussusception (7\%) and abdominal cocoon (4\%). Gastrointestinal perforations were seen in 24 patients, majority of which were neoplastic and spontaneous in origin.

\section{Reliable features of bowel obstruction}

The CT presents a gamut of imaging features ranging from non-specific such as a dilated loop of $>2.5 \mathrm{~cm}$ and small bowel feces sign (small bowel) and dilated loop $(>6-8 \mathrm{~cm}$ ) (large bowel) to features specific for cause such as swirl sign (volvulus), concertina- like configuration of bowel loops (abdominal cocoon) and sac like configuration of loops (internal hernia).

\section{Reliable features of bowel perforation}

The diagnosis of GI tract perforation is based on the direct CT findings, such as discontinuity of the bowel wall and the presence of extraluminal air, and on the indirect CT findings, such as bowel wall thickening, abnormal bowel wall enhancement and dirty fat sign (localized surrounding inflammation).

\section{DISCUSSION}

Our study group comprised of 100 patients, who presented in surgical emergency or OPD. All the patients had pain in abdomen while vomiting, distention and constipation/ obstipation were present in $70 \%, 82 \%$ and $56 \%$ patients respectively. In our study, 25 (25\%) patients showed adhesions as most common cause of obstruction (Table II). In 25 (25\%) patients, hernia was found as cause of obstruction on CT scan. Out of these, 4 patients had internal hernia on MDCT and was confirmed on exploratory laparotomy. The rest were obstructed indirect inguinal hernia and obstructed umbilical hernia. Obstruction secondary to infection or inflammation (16\%) such as tuberculosis, meckel's diverticulum was closely followed by malignancies (14\%). The rare causes of obstruction included volvulus (9) of which 5 were midgut (5\%) and 4 were cecal volvulus (4\%), intussusception (7\%) and abdominal cocoon was found in $4 \%$ cases, all of which were tubercular in etiology. Gastrointestinal perforations were seen in 24 patients, majority of which were caused by malignant neoplasms and spontaneous perforations.

\section{Adhesions}

Adhesions may be associated with peritonitis or be congenital or unexplained in origin and is a common cause of obstruction in 80\% of post-operative patients. SBO due to adhesions is diagnosed on CT when there is an abrupt 
change in the caliber of the bowel without any definite mass lesion, inflammation, or bowel wall thickening at the transition point with non-specific features of dilated small bowel loops $(>2.5 \mathrm{~cm})$, dilated large bowel loops $(>6-8 \mathrm{~cm})$ and small bowel feces sign (Table III).

\section{External hernias}

External hernias are abdominal or pelvic defect at sites of previous surgery or congenital defects. Indirect inguinal hernia is the most common type and is usually identified clinically. CT is useful for diagnosing occult hernias and depicting the precise site and type of hernia, its contents and associated complications.

\section{Inflammatory/infectious etiology}

\section{a) Crohn's Disease}

$\mathrm{SBO}$ can occur in the active phase due to acute inflammation causing luminal narrowing. Small bowel loops show mural stratification with target appearance and hyperemia resulting in the 'comb sign'. In the chronic phase, cicatricial stenosis of the bowel segments with fibrofatty proliferation is seen.

\section{b) Tuberculosis}

CT demonstrates asymmetric, irregular wall thickening of terminal ileum and caecum with enlargement of ileocecal valve and mesenteric adenopathy with central low attenuation areas suggestive of necrosis.

\section{c) Diverticulitis}

Acute diverticulitis is usually a result of inflammation of a colonic diverticulum. Rarely, it may be caused by metastases from breast carcinoma. Meckels diverticulum is the most common structural abnormality of gastrointestinal tract and commonly leads to inflammation and uncommonly leads to bowel obstruction. Plain radiography is usually the first step of suspected intestinal obstruction, but the CT usually accompanies it for identification of the possible cause.

\section{Volvulus:}

\section{a) Midgut volvulus ${ }^{7}$}

Midgut volvulus is an abdominal emergent situation mostly appreciated in children. However, with the increased use of CT in emergency department, midgut volvulus is increasingly recognized in adults. Malrotation of the small bowel is the major predisposing factor for midgut volvulus. On conventional radiography, non-specific findings of dilated gas/fluid filled bowel loops. Sonography reveals abnormal relationship between superior mesenteric artery (SMA) and superior mesenteric vein (SMV) (Vein located to the left of artery).CT especially multiplanar reformations (MPR) can increase both accuracy and confidence in the location of the transition zone in CT of SBO. Whirl sign, the more specific sign of volvulus produced by looping of superior mesenteric vein (SMV) around superior mesenteric artery (SMA), was shown in all the cases in our series (Figure 1 (a)).

\section{b) Colonic volvulus}

On conventional radiography, non-specific findings of dilated gas/fluid filled bowel loops in left upper quadrant or midabdomen. Whirl sign ${ }^{8}$ which is defined as twisting of mesentery and vessels around the bowel loops with low attenuating fatty mesentery and enhancing engorged vessels radiating from the twisted bowel is considered a specific sign (Figure $2(\mathrm{~d})$ ).

\section{Encapsulating peritoneal sclerosis (EPS-Abdominal cocoon)}

EPS is a diagnosis of multifactorial etiology, prime among these factors being long term peritoneal dialysis and bacterial peritoneal infection, especially tuberculosis. Abdominal radiographs are usually normal, however, calcification (frequent finding in setting of peritoneal dialysis) may be described on radiographs. On ultrasonography, a cauliflower appearance of bowel with a narrow base is invariably seen.

CT is the imaging modality of choice and with thin isotropic voxels and multiplanar reformations, it easily allows identification of thickened (usually $>2 \mathrm{~mm}$ ) contrast material enhanced encapsulating peritoneal membrane and the encapsulated clumped bowel loops (Figure $4(\mathrm{a}, \mathrm{b})$ ). In addition, CT can potentially help identify the cause of abdominal cocoon (omental granuloma in TB) as well as rare complications like bowel obstruction, perforation and gangrene (lack of normal bowel enhancement). A serpentine arrangement of dilated small bowel loops in a fixed U-shaped configuration is characteristic. EPS due to other causes and that due to TB might have overlapping histological features, however, a definite difference exist in the clinical and imaging manifestation of the two. Clinically, the incidence of acute small bowel obstruction is higher in patients with tubercular EPS. Ascites, especially interbowel ascites, is common in EPS and loculated fluid collections can be demonstrated and quantified. In EPS, there is preservation of central vascular pedicle (as opposed to internal hernia, in which pedicle is twisted, leading to early ischemia). ${ }^{9}$ These findings are analogous to findings of our study. Moreso, the demonstration of mesenteric and retroperitoneal lymphadenopathy in abdominal sections and tree-in-bud appearance in lower lung fields in thoracic sections further favours the tubercular etiology of obstruction.

\section{Internal hernia}

The clinical diagnosis of internal hernia is challenging because of their non-specific signs and symptoms as well as because of its intermittent nature due to spontaneous reduction. MDCT with its thin section axial images, high quality multiplanar reformations and 3-Dimensional images, currently is the first line imaging technique for pre-operative diagnosis of internal hernias. Paraduodenal hernias are the most common type on internal hernia, with left side being 3 times more common than right side. The most crucial condition in internal hernia is mechanical small bowel obstruction (closed loop obstruction). The intestinal closed loop shows a sac-like appearance and is extremely helpful imaging sign. Hernial orifice is demonstrated by convergence of bowel, mesenteric fat and vessels of closed loop. Inferior mesenteric vein (IMV) and ascending left colic artery are landmarks situated at antero-medial edge of fossa of landzert. With a left paraduodenal hernia, IMV is observed anterior and medial to hernia orifice and entrapped intestine $e^{10,11}$ (Figure 3 (a)). This are in congruence to findings of our study that also demonstrates a left paraduodenal hernia with the hernial sac in left anterior pararenal space and inferior 
mesenteric vein being antero-medial to it.

\section{GI tract perforation}

The diagnosis of GI tract perforation is based on the direct CT findings, such as discontinuity of the bowel wall and the presence of extraluminal air, and on the indirect CT findings, such as bowel wall thickening, abnormal bowel wall enhancement and dirty fat sign (localized surrounding inflammation) (Table IV). The major causes of a large bowel perforation are malignant neoplasm, diverticulitis and spontaneous perforation. Perforations from malignant neoplasm, spontaneous perforation, diverticulitis, blunt trauma and ischemia are frequently observed in the left colon whereas inflammatory lesions and penetrating trauma tend to occur in the right colon. The rectum and sigmoid colon are the predominant sites of iatrogenic injuries. MDCT is useful to identify discontinuity of the bowel wall (Figure 5 (b)), especially when the axial CT images are indeterminate. MDCT is highly sensitive in determining the site of perforation. Free air confined in the intrahepatic fissure of ligamentum teres and a large amount of intraperitoneal air is commonly due to gastro-duodenal perforation. Air in the lesser sac usually indicates perforation of stomach or duodenum or less frequently, rupture of the lower esophagus or transverse colon. Air trapped in the mesenteric folds is commonly due to perforation of the colon and small bowel. If free air is present only in the pelvis, the likely site of perforation is the colon or, less commonly, the small bowel. On radiography, extensive extraluminal air suggestive of perforation was seen. CT also allows grading of amount of perforation based on amount of ascites and extent of inflammatory changes adjacent to the GI tract wall on CT (mild- maximum extension of dirty fat sign $<5 \mathrm{~cm}$, moderate$5-10$, severe- > $10 \mathrm{~cm}) .^{12,13,14,15}$ In our study, neoplastic bowel wall thickening was seen in distal transverse colon and perforations in cecum and transverse colon proximal to thickening were seen.(Figure 5 (a),(b)).

\section{CONCLUSION}

We conclude that MDCT is highly sensitive and specific in determining the presence of bowel obstruction and attains prime diagnostic value when it comes to diagnosis of obstruction due to uncommon causes like volvulus, encapsulating peritoneal sclerosis and internal hernias. CT can provide important information for the diagnosis of gastrointestinal tract perforation, especially in aspect of evaluating the site of perforation. Therefore, MDCT is an indispensable radiological tool in evaluating bowel obstruction and perforation irrespective of the etiology. Moreso, it assumes special importance when the underlying etiology is rare.

\section{REFERENCES}

1. Paulson EK, Thompson WM. Review of small-bowel obstruction: the diagnosis and when to worry. Radiology. 2015;275(2):332-42.

2. Jaffe T, Thompson WM. Large-bowel obstruction in the adult: classic radiographic and CT findings, etiology, and mimics. Radiology. 2015;275(3):651-63.

3. Silva AC, Pimenta M, Guimaraes LS. Small bowel obstruction: what to look for. Radiographics. 2009;29(2):423-39.

4. Kim SH, Shin SS, Jeong YY, Heo SH, Kim JW, Kang HK. Gastrointestinal tract perforation: MDCT findings according to the perforation sites. Korean journal of radiology. 2009;10(1):63-70.

5. Saini DK, Chaudhary P, Durga CK, Saini K. Role of multislice computed tomography in evaluation and management of intestinal obstruction. Clinics and practice. 2013;3(2).

6. Hodel J, Zins M, Desmottes L, Boulay-Coletta I, Jullès MC, Nakache JP, Rodallec M. Location of the transition zone in CT of small-bowel obstruction: added value of multiplanar reformations. Abdominal imaging. 2009;34(1):35-41.

7. Peterson CM, Anderson JS, Hara AK, Carenza JW, Menias CO. Volvulus of the gastrointestinal tract: appearances at multimodality imaging. Radiographics. 2009;29(5):1281-93.

8. Frank AJ, Goffner LB, Fruauff AA, Losada RA. Cecal volvulus: the CT whirl sign. Abdominal imaging. 1993;18(3):288-9.

9. Singhal M, Krishna S, Lal A, Narayanasamy S, Bal A, Yadav TD, Kochhar R, Sinha SK, Khandelwal N, Sheikh AM. Encapsulating Peritoneal Sclerosis: The Abdominal Cocoon. RadioGraphics. 2018;39(1):62-77.

10. Doishita S, Takeshita T, Uchima Y, Kawasaki M, Shimono T, Yamashita A, Sugimoto M, Ninoi T, Shima $\mathrm{H}$, Miki Y. Internal hernias in the era of multidetector CT: correlation of imaging and surgical findings. Radiographics. 2015;36(1):88-106.

11. Trigui A, Guirat A, Rejab H, Kardoun N, Frikha F, Amar MB. An Uncommon Cause of Acute Bowel Obstruction: The Left Para. Duodenal Hernia. Nigerian Journal of Surgery. 2012;18(2):97-9.

12. Hainaux B, Agneessens E, Bertinotti R, De Maertelaer V, Rubesova E, Capelluto E, Moschopoulos C. Accuracy of MDCT in predicting site of gastrointestinal tract perforation. American Journal of Roentgenology. 2006;187(5):1179-83.

13. Ghekiere O, Lesnik A, Millet I, Hoa D, Guillon F, Taourel P. Direct visualization of perforation sites in patients with a non-traumatic free pneumoperitoneum: added diagnostic value of thin transverse slices and coronal and sagittal reformations for multi-detector CT. European radiology. 2007;17(9):2302-9.

14. Furukawa A, Sakoda M, Yamasaki M, Kono N, Tanaka T, Nitta N, Kanasaki S, Imoto K, Takahashi M, Murata K, Sakamoto T. Gastrointestinal tract perforation: CT diagnosis of presence, site, and cause. Abdominal imaging. 2005;30(5):524-34.

15. Del Gaizo AJ, Lall C, Allen BC, Leyendecker JR. From esophagus to rectum: a comprehensive review of alimentary tract perforations at computed tomography. Abdominal imaging. 2014;39(4):802-23.

Source of Support: Nil; Conflict of Interest: None

Submitted: 07-12-2019; Accepted: 02-01-2020; Published online: 11-02-2020 